\title{
Oxidative Stress and COPD: The Impact of Oral Antioxidants on Skeletal Muscle Fatigue
}

\author{
Matthew J. Rossman ${ }^{1,2}$, H. Jonathan Groot ${ }^{1,2}$, Van Reese ${ }^{1,3}$, Jia Zhao ${ }^{1,3}$, Markus \\ Amann $^{1,2,3}$, and Russell S. Richardson ${ }^{1,2,3}$ \\ ${ }^{1}$ Geriatric Research, Education, and Clinical Center George E. Whalen VA Medical Center Salt \\ Lake City, Utah \\ ${ }^{2}$ Department of Exercise and Sport Science University of Utah Salt Lake City, Utah \\ ${ }^{3}$ Department of Internal Medicine, Division of Geriatrics University of Utah Salt Lake City, Utah
}

\begin{abstract}
PURPOSE-Oxidative stress may contribute to exercise intolerance in patients with chronic obstructive pulmonary disease (COPD). This study sought to determine the effect of an acute oral antioxidant cocktail (AOC: vitamins $\mathrm{C}, \mathrm{E}$, and alpha-lipoic acid) on skeletal muscle function during dynamic quadriceps exercise in COPD.

METHODS-Ten patients with COPD performed knee extensor exercise to exhaustion and isotime trials following either the AOC or placebo (PL). Pre- to post-exercise changes in quadriceps maximal voluntary contractions (MVCs) and potentiated twitch forces $\left(\mathrm{Q}_{\mathrm{tw}, \mathrm{pot}}\right)$ quantified quadriceps fatigue.
\end{abstract}

RESULTS-Under PL conditions, the plasma electron paramagnetic resonance (EPR) spectroscopy signal was inversely correlated with the forced expiratory volume in one second to forced vital capacity ratio $\left(\mathrm{FEV}_{1} / \mathrm{FVC}\right)$, an index of lung dysfunction ( $\left.\mathrm{r}=-0.61, \mathrm{p}=0.02\right)$, and $\mathrm{MVC}$ force $(\mathrm{r}=-0.56, \mathrm{p}=0.04)$. AOC consumption increased plasma ascorbate levels $(10.1 \pm 2.2$ to $24.1 \pm 3.8 \mathrm{ug} / \mathrm{ml}, \mathrm{p}<0.05)$ and attenuated the area under the curve of the EPR spectroscopy free radical signal ( $11.6 \pm 3.7$ to $4.8 \pm 2.2 \mathrm{AU}, \mathrm{p}<0.05)$, but did not alter endurance time or quadriceps fatigue. The ability of the AOC to decrease the EPR spectroscopy signal, however, was prominent in those with high basal free radicals ( $\mathrm{n}=5$, PL: $19.7 \pm 5.8$ to AOC: $5.8 \pm 4.5$ AU, $\mathrm{p}<0.05$ ) with minimal effects in those with low levels ( $n=5$, PL: $1.6 \pm 0.5$ to AOC: $3.4 \pm 1.1 \mathrm{AU}$ ).

DISCUSSION-These data document a relationship between directly measured free radicals and lung dysfunction, and the ability of the AOC to decrease oxidative stress in COPD. Acute amelioration of free radicals, however, does not appear to impact dynamic quadriceps exercise performance.

\section{Keywords}

Free radicals; electron paramagnetic resonance spectroscopy; peripheral fatigue; knee extensor

Corresponding Author: Russell S. Richardson VA Medical Center Bldg 2, Rm 1D25 500 Foothill Drive Salt Lake City, UT 84148 801.582.1565 Ext. 4344 Fax 801.584.5658 r.richardson@ @sc.utah.edu.

Conflicts of Interest: None. 


\section{INTRODUCTION}

Oxidative stress, which can be defined as an imbalance between oxidant and antioxidant forces in favor of the former (33), is prevalent in patients with COPD $(12,13)$. Numerous factors contribute to the elevated oxidant burden in this population, but the most accepted source of free radicals is the inflammatory pathology of the lung disease (39). In addition, exhaustive knee extensor exercise, which minimally increases ventilation (37), has been documented to increase markers of oxidative damage, such as plasma lipid peroxidation products, in patients with COPD but not in healthy controls (12). Inhibition of the superoxide generator xanthine oxidase during exercise in patients with COPD ameliorated the increase in lipid peroxidation products and prevented an increase in the ratio of oxidized to reduced glutathione (19). Therefore, exercising skeletal muscle has been implicated as a source of free radicals that contribute to oxidative stress in this population.

Pulmonary system abnormalities certainly limit exercise in patients with COPD (3); however, peripheral muscle abnormalities also negatively impact exercise performance and activities of daily living (36). Indeed, significant peripheral locomotor muscle fatigue occurs in patients with COPD following cycle exercise (3), and the perception of leg fatigue is frequently recognized as the primary symptom contributing to exercise intolerance (21). Therefore, enhancing quality of life and exercise capacity in COPD through peripheral muscle therapy is regarded as an important rehabilitative tool (26). In the context of skeletal muscle function, free radicals both depress muscle force production (32) and exaggerate the discharge frequency of thin-fiber muscle afferents (15), which contribute to central fatigue (2). Therefore, targeting oxidative stress in COPD to attenuate the development of fatigue and improve exercise tolerance appears to be a reasonable theory.

$\mathrm{N}$-acetylcysteine (NAC) is a glutathione precursor and a potent pharmacological antioxidant. In patients with COPD, NAC has been documented to improve quadriceps exercise endurance and lessen markers of oxidative damage at exhaustion (24). NAC however, also appears to improve both small muscle mass (34) and whole body (27) exercise tolerance in young, healthy individuals, which suggests that the benefits of NAC may not be unique to a cohort with a heightened susceptibility to oxidative stress. In addition, NAC administration can be accompanied by lightheadedness and nausea (34), and these adverse side effects potentially limit the efficacy of this antioxidant as a therapeutic intervention. There is currently little known about the potential of other antioxidant interventions to improve exercise tolerance in patients with COPD.

Our group has previously documented the ability of an orally administered, readily available, antioxidant cocktail (AOC; vitamin $\mathrm{C}$, vitamin $\mathrm{E}$, and a-lipoic acid) to reduce carbon and $\mathrm{O}_{2}$-centered free radical levels, as measured by EPR spectroscopy in the elderly (43), and have beneficial vascular effects in patients with COPD (18). Therefore, this study sought to examine the effects of this AOC on free radical concentration and skeletal muscle fatigue following single-leg dynamic knee extensor exercise in patients with COPD. We tested the hypotheses that in patients with COPD, the AOC would: 1) raise antioxidant levels and decrease free radicals, as assessed by plasma ascorbate concentration and electron paramagnetic resonance (EPR) spectroscopy, 2) improve knee extensor endurance exercise time to exhaustion, and 3) attenuate the magnitude of peripheral quadriceps fatigue assessed following knee extensor exercise matched for intensity and duration (isotime). 


\section{METHODS}

\section{Subjects}

Written, informed consent was obtained from all participants prior to their inclusion in this study, and the Institutional Review Boards of the University of Utah and the Salt Lake City VA Medical Center approved all protocols. Ten patients with COPD were recruited for the study based on spirometric evidence of moderate to severe airflow obstruction ( $\mathrm{FEV}_{1}<80 \%$ predicted, $\mathrm{FEV}_{1} / \mathrm{FVC} \leq 0.7$ (10)), as assessed by standard pulmonary function tests performed during an initial visit to the laboratory. General morphometric characteristics, including thigh volume, which was used to estimate quadriceps muscle mass (17), were also determined during this visit. In addition, to ensure supramaximality of stimulation during magnetic stimulation of the femoral nerve (described below), the plateau in evoked force following serial twitch forces, obtained every 30 seconds, at 70, 80, 85, 90, 95, and $100 \%$ of maximal stimulator output, was also evaluated. To add to these subject characteristics, data from a parallel study involving arterial blood samples, in which 9 of the 10 current subjects took part, are also presented in this study.

\section{Exercise Protocol}

All subjects were familiarized with knee extensor exercise, which was performed at a cadence of $60 \mathrm{rpm}$, during two visits to the laboratory. Subsequently, peak knee extensor work rate was determined with subject specific protocols designed to reach exhaustion within 8-12 minutes, consisting of a 2-5 W/min increases. Subjects then performed two practice constant load exercise trials at $80 \%$ of maximal workload to the limit of tolerance ( $\mathrm{T}_{\text {LIM }}$; task failure was defined as a fall below $50 \mathrm{rpm}$ ). Next, in a single blind, repeatedmeasures design, $\mathrm{T}_{\mathrm{LIM}}$ trials were performed following AOC or PL administration, with the first and second doses consumed 1.5 hours and 1-hour prior to each $\mathrm{T}_{\mathrm{LIM}}$ trial. This regimen was repeated with the opposite condition after at least 48 hours to allow recovery and treatment washout, such that all subjects performed $\mathrm{T}_{\mathrm{LIM}}$ trials under both AOC and PL conditions in a counter-balanced manner. The shorter of two $\mathrm{T}_{\mathrm{LIM}}$ times was then matched with the opposite condition (AOC or PL) on a successive visit to allow isotime comparisons. Thus, all patients completed three exercise trials during data collection (see Figure, Supplemental Digital Content 1, a schematic of the protocol).

Neuromuscular function tests were performed before and 10 minutes after the $\mathrm{T}_{\mathrm{LIM}}$ and isotime trials. In addition, venous blood samples were taken before and immediately after the $\mathrm{T}_{\mathrm{LIM}}$ and isotime trials to determine pro- and antioxidant status, and for spin trapping and EPR spectroscopy to directly assess free radical concentration. One minute of resting data were collected and subjects performed one minute of unloaded warm-up knee extensor exercise prior to each exercise bout. Ventilation, gas exchange, heart rate, ratings of perceived exertion and breathlessness, arterial oxygen saturation, femoral blood velocity and quadriceps electromyograms (EMG) were measured throughout all trials.

\section{Antioxidant supplementation}

The AOC was administered in a split dose to improve absorption and maintain the time of antioxidant efficacy, with the first dose consisting of $300 \mathrm{mg}$ a-lipoic acid, $500 \mathrm{mg}$ vitamin $\mathrm{C}$ and $200 \mathrm{IU}$ of vitamin E, consumed 1.5 hours prior to the exercise testing, and the second dose consisting of the same amounts of a-lipoic acid and vitamin C and $400 \mathrm{IU}$ of vitamin $\mathrm{E}$ consumed $1 \mathrm{hr}$ prior to the testing. PL microcrystalline cellulose capsules of similar taste, color, and appearance were likewise consumed in 2 doses within the same time frame (41). This AOC, and the dosing strategy employed, has been previously documented to reduce carbon and $\mathrm{O}_{2}$-centered free radical levels as measured by EPR spectroscopy in the elderly (43), and improve vascular function in patients with COPD (18). Subjects were blinded to 
the experimental condition (AOC or PL), as well as all but one member of the research team, in a single blind design. Due to the intense and exhaustive nature of the exercise trials, patients were not fasted during the supplementation, but were instructed to consume a similar, benign, low-fat meal 3-4 hours prior to data collection on all study days.

\section{Oxidative stress, antioxidant assays, and free radicals}

Plasma samples were stored at $-80^{\circ} \mathrm{C}$ until analysis. Lipid peroxidation, a marker of oxidant damage, was assessed by thiobarbituric acid reactive substances (TBARS) (44) (Bioassays Systems, Hayward, CA) as well as malondialdehyde levels (Bioxytech LPO-586, Foster City, CA) and lipid hydroperoxides (Cayman Chemical Company, Ann Arbor, MI). Total antioxidant capacity was assessed by determining the ferric reducing ability of plasma (FRAP), using the method described by Benzie and Strain (7). The efficacy of the AOC specific to plasma ascorbate levels was assayed as previously described (9) (CosmoBio, Carlsbad, CA). Endogenous antioxidant activity, assessed by superoxide dismutase and catalase activity, was also assayed in the plasma (40) (Cayman Chemical Company, Ann Arbor, MI).

EPR spectroscopy was performed on pre- and postexercise blood samples to directly assess the ability of the AOC to reduce the concentration of free radicals, as previously described (35). Briefly, $3 \mathrm{ml}$ of venous blood was collected into a vacutainer containing $1 \mathrm{ml}$ of the spin trap a-phenyl-tert-butylnitrone $(0.0140 \mathrm{~mol} / \mathrm{l})$. After centrifugation, the a-phenyl-tertbutylnitrone adduct $(200 \mu \mathrm{l})$ was pipetted into a precision-bore quartz EPR sample tube (Wilmad, Vineland, NJ). EPR spectroscopy was then performed at $21{ }^{\circ} \mathrm{C}$ using an EMX Xband spectrometer (Bruker, MA) and commercially available software (version 2.11, Bruker Win EPR System), which was also used to calculate the area under the curve of the EPR spectroscopy signal by double integration.

\section{Ventilation, gas exchange, heart rate, and perceived exertion}

Ventilation and pulmonary gas exchange were measured at rest and during exercise with a metabolic cart (ParvoMedics, Sandy, UT). Heart rate, determined from the R-R interval of a three-lead electrocardiogram, and arterial oxygen saturation estimated with a pulse oximeter (Nellcor N-595, Pleasanton, CA, USA) with forehead sensors, were acquired at $200 \mathrm{~Hz}$ using a data acquisition system (AcqKnowledge; Biopac Systems, Goleta, CA). Ratings of perceived exertion were taken every minute during the exercise trials using Borg's CR10 scale (8).

\section{Leg blood flow}

Measurements of femoral artery blood velocity and vessel diameter in the leg being studied were performed at rest and throughout exercise, using a Logic 7 ultrasound system (General Electric Medical Systems, WI, USA) as previously described (38). Briefly, arterial diameter was measured, and mean velocity values (angle-corrected, and intensity weighted areaunder-the-curve) were calculated using commercially available software (Logic 7). Using arterial diameter and mean velocity, blood flow in the femoral artery was calculated as: blood flow $=($ mean velocity $) \pi(\text { vessel diameter } / 2)^{2} \times 60$.

\section{Quadriceps electromyograms}

Quadriceps electromyograms (EMG) were recorded from the vastus lateralis muscle during exercise from electrodes placed in a bipolar configuration over the middle of the muscle belly, with the active electrodes placed over the motor point of the muscle and the reference electrode in an electrically neutral site (2). Raw EMG signals were filtered with a bandpass filter (with a low pass cut-off frequency of $15 \mathrm{~Hz}$ and a high pass cut-off frequency of 650 
$\mathrm{Hz}$ ) and after visual inspection of the filtered signal; a threshold voltage was set to identify the onset of EMG activity (AcqKnowledge; Biopac Systems, Goleta, CA). For data analysis, the integral of each EMG burst (integrated EMG) was calculated to determine the percent increase in integrated EMG from the first minute of exercise (2), an index of the development of peripheral fatigue during exercise. The EMG electrodes were also used to record magnetically evoked compound action potentials ( $\mathrm{M}$-waves) to evaluate changes in membrane excitability from pre- to post-exercise $\mathrm{Q}_{\mathrm{tw}, \text { pot }}$ assessments.

\section{Neuromuscular function assessment}

The magnitude of peripheral quadriceps fatigue was quantified by the pre- to post-exercise changes in quadriceps MVC and $Q_{t w, p o t}$ evoked by supramaximal magnetic stimulation of the femoral nerve $(2,31)$. Specifically, while subjects lay semi-recumbent in the knee extensor chair with a knee joint angle of 90 degrees, subjects performed a series of six MVCs separated by 30 seconds, with $\mathrm{Q}_{\mathrm{tw} \text {,pot }}$ assessments interspersed 5 seconds after each MVC, before and 10 minutes after each exercise trial. In addition, to quantify voluntary activation of the quadriceps during the MVCs, the additional force generated by a single twitch superimposed on the MVC was compared with the force produced by the potentiated twitch immediately following the MVC to determine the percent voluntary muscle activation (2). Force was obtained from a calibrated load cell (Transducer Techniques, Temecula, CA) connected to a non-compliant strap placed around the subject's ankle and acquired at $200 \mathrm{~Hz}$ with a data acquisition system (AcqKnowledge; Biopac Systems, Goleta, CA). A magnetic stimulator (Magstim 200, The Magstim Company Ltd, Wales UK) connected to a double 70 $\mathrm{mm}$ coil was used to stimulate the femoral nerve,. Peak force, maximal rate of force development and maximal relaxation rate were analyzed for all $\mathrm{Q}_{\mathrm{tw} \text {,pot }}$ values (25).

\section{Statistical Analysis}

Two-way repeated measures ANOVA were used to compare the effect of antioxidant treatment on physiological parameters during exercise, with a Tukey post-hoc analysis if a significant main effect was found. Student's paired $t$ tests were used to compare the effect of the AOC in terms of antioxidant efficacy, end exercise physiological parameters, and indices of peripheral fatigue. Correlations between variables were evaluated using Pearson correlation coefficients. Statistical significance was set at $\alpha=0.05$ for all tests. All group data are expressed as means \pm standard error of the mean.

\section{RESULTS}

\section{Subject Characteristics}

Ten subjects completed all exercise trials, whose data are reflected in Table 1. Data obtained in the parallel study on the same subjects $(n=9)$ involving arterial blood samples are also presented in Table 1. One subject was a current smoker who was able for refrain from smoking for 12 hours prior to data collection. Interestingly, resting free radical concentration as assessed by EPR spectroscopy of the pre-exercise blood samples under PL conditions, indicative of the basal free radical load, was inversely correlated with $\mathrm{FEV}_{1} / \mathrm{FVC}(\mathrm{r}=-0.65$, $\mathrm{p}=0.02)$ and baseline MVC force $(\mathrm{r}=-0.56, \mathrm{p}=0.04)($ Figure 1$)$.

\section{Antioxidant Efficacy}

Consumption of the AOC increased plasma ascorbate levels and reduced the EPR spectroscopy signal AUC (Figure 2), but did not alter any other markers of oxidative stress (TBARS: $5.1 \pm 0.3$ vs $4.7 \pm 0.3 \mathrm{uM}$, malondialdehyde: $1.2 \pm 0.1$ vs $1.1 \pm 0.1 \mathrm{uM}$, lipid hydroperoxides: $0.16 \pm 0.01$ vs $0.15 \pm 0.02 \mathrm{nM}$ ) or antioxidant status (FRAP: $0.99 \pm 0.1$ vs $1.0 \pm 0.1$ superoxide dismutase: $21.4 \pm 5.5$ vs $20.5 \pm 5.3 \mathrm{U} / \mathrm{ml}$, catalase: $22.4 \pm 2.8$ vs $27.7 \pm 5.0$ 
$\mathrm{nmol} / \mathrm{min} / \mathrm{ml}$, PL vs AOC respectively) prior to exercise. Following exercise, FRAP was increased above the PL condition with the AOC $(1.0 \pm 0.1$ vs $1.1 \pm 0.1 \mathrm{mM}, \mathrm{p}<0.05$, respectively), however, there were no other significant pre- to postexercise changes.

\section{Performance Trials}

Exercise to exhaustion resulted in a decrease in quadriceps muscle function, as illustrated by a fall in all measured indices of fatigue $\left(\mathrm{MVC}, \mathrm{Q}_{\mathrm{tw}, \text { pot }}\right.$, maximal rate of force development, and maximal relaxation rate) and a similar increase in the integrated EMG signal between trials (AOC: $108.5 \pm 17.4 \%$, PL: $107.8 \pm 17.1 \%$ ). M-wave characteristics were maintained from pre to postexercise and were not different between trials, indicating preserved membrane excitability. In addition, the percent voluntary muscle activation was reduced from pre- to postexercise following both exercise trials, but was not different between conditions (AOC: $-5.3 \pm 1.2 \%$, PL: $-5.1 \pm 1.7 \%$ ). Thus, AOC consumption did not alter the physiological responses to exercise, and both trials resulted in a similar time to exhaustion and magnitude of end-exercise fatigue (Figure 3). The change in endurance time from the PL trial following ingestion of the AOC was also unrelated, by correlational analysis, to the change in free radical concentration assessed by EPR spectroscopy $(r=0.4, p>0.05)$.

\section{Isotime Trials}

Following exercise of equal duration $(7.1 \pm 0.7 \mathrm{~min})$ and intensity $(23 \pm 3 \mathrm{~W})$, similar levels of end exercise quadriceps fatigue were attained with either AOC or PL consumption (Figure $3)$. The change in quadriceps fatigue with the AOC compared to the PL trial was also unrelated, by correlational analysis, to the change in free radical concentration assessed by EPR spectroscopy $(\mathrm{r}=-0.1, \mathrm{p}>0.05)$. M-wave characteristics were unchanged by the isotime trials and the percent voluntary muscle activation was reduced from pre-exercise values to a similar extent in both conditions (AOC: $-4.1 \pm 0.8 \%$, PL: $-3.5 \pm 0.7 \%$ ). The physiological responses to exercise (gas exchange, ventilation, heart rate, arterial oxygen saturation, integrated EMG, rating of perceived exertion, and femoral artery blood flow) were not different between conditions and are documented in Figure 4.

\section{DISCUSSION}

In this study, we documented the ability of an oral AOC (vitamins C, E and alpha-lipoic acid) to decrease the resting plasma free radical concentration in patients with COPD, as assessed by EPR spectroscopy. We also observed a broad array of resting free radical levels, in line with the diverse etiology of COPD. The resting free radical level was inversely correlated with the $\mathrm{FEV}_{1} / \mathrm{FVC}$ ratio, which is an index of the degree of lung dysfunction. Resting free radical levels were also inversely correlated with baseline MVC force. The correlational relationships suggest that patients with more significant disease progression tend to have higher resting plasma free radicals. However, despite clearly documented antioxidant effects, we did not observe any functional consequences in terms of knee extensor exercise endurance time or the rate of development of peripheral muscle fatigue. Collectively, this study suggests that susceptibility to oxidative stress may be related to pulmonary disease severity and that administration of the AOC may acutely depress plasma free radicals in patients with COPD. Acutely decreasing free radicals, however, does not necessarily impact dynamic knee extensor exercise performance in patients with COPD.

\section{Oxidative stress and lung disease}

Oxidative stress may pathophysiologically alter redox sensitive inflammation, mitochondrial function, and myofilament interactions in patients with $\operatorname{COPD}(13,23)$. Indeed, elevations in oxidative stress in patients with COPD have been extensively documented systemically (12), and in the lung $(6,28)$. Specifically, Barreiro et al $(6)$ recognized higher protein carbonyl 
groups, a marker of protein oxidation, in diaphragm muscle samples of patients with severe COPD. Barreiro et al (6) also documented an inverse relationship between protein carbonyls and $\mathrm{FEV}_{1}(\mathrm{r}=-0.734, \mathrm{p}<0.01)$ in the patients with moderate to severe airflow obstruction. Similar negative correlations between other markers of oxidative damage and airflow limitation have also been observed in the exhaled breath condensate (28) as well as in the blood (29). However, EPR spectroscopy is considered to be the most sensitive technique for the direct detection of free radicals (5), and while EPR spectroscopy has been employed previously in the bronchoalveolar lavage fluid (30), to our knowledge, the current study is the first to use the technique to directly assay the concentration of oxygen- and carboncentered free radicals in the plasma of patients with COPD, reflective of the systemic free radical concentration.

In this study, resting free radical concentration was inversely correlated with $\mathrm{FEV}_{1} / \mathrm{FVC}(\mathrm{r}=$ $-0.65, p=0.02$, Figure 1). An inverse relationship between oxidative stress and lung function is in accord with previous literature, and corroborates the role of oxidative stress in the pathology of COPD. Measured resting free radical concentration was also negatively correlated with baseline MVC force $(r=-0.56, p=0.04$, Figure 1$)$, implying a detrimental relationship between systemic free radical load and skeletal muscle function. AOC administration increased plasma ascorbate levels and decreased the concentration of plasma free radicals, assessed by EPR spectroscopy (Figure 2). However, upon secondary analysis of the individual responses presented as average data in Figure 2, a dichotomous response to the AOC was observed (Figure 5). Specifically, ingestion of the AOC caused a universal increase in plasma ascorbate, but free radicals were only decreased in patients with high basal free radicals in the PL condition. In contrast, AOC consumption had minimal effects in patients with low basal free radicals in the PL condition. Moreover, the inverse relationship between plasma free radicals and the $\mathrm{FEV}_{1} / \mathrm{FVC}$ ratio suggests that elevated oxidative stress may only be of significant concern in patients with a greater degree of pulmonary dysfunction. Collectively, these data support a role for the AOC to decrease plasma free radicals in patients with COPD when basal free radical level is high, which may primarily occur with significant disease progression.

\section{Performance time and oxidative stress}

Oxidative stress may modulate exercise performance in both health and disease (32). Koechlin et al (24) documented an improvement in quadriceps exercise time to exhaustion following oral NAC administration in patients with COPD. The effects of NAC, however, are not unique to patients with COPD: ergogenic effects have also been documented in young, healthy subjects $(27,34)$. Favorable effects, however, are typically only demonstrated following intravenous administration, which can yield greater plasma NAC levels compared to oral administration. In contrast, beneficial effects on exercise performance have not typically been demonstrated with vitamins $\mathrm{C}$, E, or alpha lipoic acid $(4,32)$. However, the current study, to our knowledge, is the first to assess the potential for non-pharmacological doses of vitamins $\mathrm{C}, \mathrm{E}$, and alpha lipoic acid in modulating exercise tolerance in patients with COPD.

AOC administration did not change endurance time to exhaustion (Figure 3). The lack of effect of the AOC in comparison to NAC was surprising given the striking similarities between the characteristics of the subjects in the study by Koechlin et al (24) and the current study cohort (i.e. moderate to severe COPD and an average age of 62 yrs of age). The ergogenic effect demonstrated in the Koechlin et al (24) study may potentially be explained by the four-day NAC loading period, enhancing the ability for NAC to enter the skeletal muscle itself. The primary aim of the current study, however, was to examine the impact of an acute, oral AOC on skeletal muscle function during dynamic exercise in patients with COPD. Acute administration of this AOC has no known side effects and therefore has the 
potential to impact rehabilitative medicine in this population. Thus, in an attempt to reconcile the discordant responses between our study and the Koechlin et al study (24), and in light of our AOC efficacy data (Figure 5), we attempted to correlate the change in free radical concentration following AOC consumption with the change in endurance time, but did not find evidence of a relationship $(r=0.4, p>0.05)$. Therefore, these data suggest that acutely decreasing free radicals in patients with COPD with the AOC does not improve high-intensity, dynamic knee extensor exercise time to exhaustion.

\section{Oxidative Stress and Fatigue}

Oxidant production by exercising skeletal muscle has been suggested to negatively impact exercise capacity in patients with COPD $(12,13)$. Indeed, Couillard et al (12) have previously documented an inverse relationship between the exercise-induced increase in muscle oxidative stress and quadriceps endurance time in patients with COPD. In the current study, we employed isotime exercise to allow comparisons of the magnitude of end-exercise quadriceps fatigue following identical exercise bouts with the PL and the AOC. Isotime trials are, in general, less variable than the performance trials and as illustrated in Figure 4, the physiological responses to the isotime exercise bouts in this study were remarkably similar. The percent increase in the integrated EMG signal represents the development of peripheral fatigue during knee extensor exercise, and the integrated EMG response was one of the nearly identical physiological responses assessed in both trials (Figure 4).

Accordingly, no differences in end-exercise peripheral fatigue were observed (Figure 3) and there was no relationship between the change in free radical concentration and the change in quadriceps fatigue following AOC consumption $(\mathrm{r}=-0.1, \mathrm{p}>0.05)$. Therefore, although the negative relationship between basal free radical level and MVC force (Figure 1) implies a detrimental relationship between chronic oxidative stress and muscle function in patients with COPD, acute amelioration of this oxidant load does not diminish the magnitude of endexercise quadriceps fatigue following dynamic knee extensor exercise.

\section{Blood flow, vascular function, oxygen delivery and oxidative stress}

The preponderance of efficacy data for this AOC is derived from studies examining vascular function in populations predisposed to oxidative stress $(16,35,42)$. Our group has documented that the AOC can restore vascular function in the elderly $(18,41)$ and patients with COPD (18) as assessed by flow-mediated dilation and submaximal handgrip exercise (16). In addition, this AOC has been observed to enhance end-exercise muscle perfusion and improve muscle oxidative capacity in the elderly during plantar flexion exercise (42). Oxidative stress is elevated in COPD $(11,12,28)$, so it was hypothesized that the vascular effects of the AOC would be more pronounced. Admittedly, evidence for improved vascular function altering exercising muscle blood flow is relatively sparse. However, it is possible that reducing oxidative stress may augment nitric oxide bioavailability and increase skeletal muscle blood flow $(14,22)$. Enhancing blood flow and potentially oxygen delivery would likely improve exercise performance (1). Thus, in the current study, femoral artery blood flow was measured during knee extensor exercise; however, no difference between the PL and AOC trials was observed (Figure 4). Similar hemodynamic responses between conditions suggest similar oxygen delivery to the working muscle. It is possible, however, that the varied basal free radical concentration and the subsequent heterogeneous effects of the AOC (Figure 5) may have obscured any differences in skeletal muscle blood flow.

\section{Limitations}

It is important to acknowledge several limitations of the current study. Most notably, the timing and acute nature of the antioxidant administration $(\sim 1.5$ and $\sim 2$ hours prior to the preand post-exercise blood draws, respectively) may have precluded detecting any changes in the indirect markers of oxidative damage (TBARs, malondialdehyde levels, and lipid 
hydroperoxides). Indeed, Koechlin et al (24) documented an increase in plasma TBARs only six hours after exhaustive quadriceps exercise in patients with COPD; the plasma TBARs measured immediately after exercise were not different from pre-exercise levels. In addition, indirect markers of oxidative stress, such as TBARs and malondialdehyde levels, are generally regarded as insensitive markers of lipid peroxidation (20). However, to gain a comprehensive understanding of the oxidant/antioxidant balance, the current study quantified antioxidant capacity, indirect markers of oxidative damage, as well as directly measuring free radicals, as suggested by Jackson et al. (20). Collectively, these data support an acute antioxidant effect of the AOC in patients with COPD, which was most prominently demonstrated by the increase in ascorbate and decrease in the EPR spectroscopy free radical signal, as well as an increase in the post-exercise FRAP value. It is also possible that a greater dose or different antioxidant administration strategy may be necessary for antioxidant effects to occur in the muscle itself. The ease of administration and acute nature of the antioxidant strategy of the current study, however, represents an important first step in the evaluation of antioxidant administration, oxidative stress, and exercise performance in COPD.

\section{Conclusion}

This study reveals a relationship between the degree of lung dysfunction in patients with COPD and resting free radical concentration, as assessed by EPR spectroscopy measurements in blood. Resting free radical concentration was also inversely related to quadriceps MVC force. Collectively, these relationships suggest that patients with more advanced COPD tend to have higher free radical levels, and high free radicals may have a detrimental effect on skeletal muscle force production. Additionally, the ability of an oral AOC to decrease oxidative stress has been documented in patients with COPD. However, the translation of decreased oxidative stress to improved knee extensor exercise performance and attenuated muscle fatigability is not supported by the current data. Thus, while a potentially beneficial role for the AOC can be inferred for patients with COPD, an acute decrease in free radicals does not appear to be related to knee extensor exercise endurance performance.

\section{Supplementary Material}

Refer to Web version on PubMed Central for supplementary material.

\section{Acknowledgments}

The authors would like to thank the subjects for their gracious participation and are thankful for financial support by the National Institutes of Health grant PO1 HL 09830.

The results of the present study do not constitute endorsement by ACSM.

\section{References}

1. Amann M, Calbet JA. Convective oxygen transport and fatigue. J Appl Physiol. 2008; 104(3):86170. [PubMed: 17962570]

2. Amann M, Proctor LT, Sebranek JJ, Pegelow DF, Dempsey JA. Opioid-mediated muscle afferents inhibit central motor drive and limit peripheral muscle fatigue development in humans. J Physiol. 2009; 587(Pt 1):271-83. [PubMed: 19015193]

3. Amann M, Regan MS, Kobitary M, Eldridge MW, Boutellier U, Pegelow DF, Dempsey JA. Impact of pulmonary system limitations on locomotor muscle fatigue in patients with COPD. Am J Physiol Regul Integr Comp Physiol. 2010; 299(1):R314-24. [PubMed: 20445160] 
4. Ashton T, Young IS, Peters JR, Jones E, Jackson SK, Davies B, Rowlands CC. Electron spin resonance spectroscopy, exercise, and oxidative stress: an ascorbic acid intervention study. J Appl Physiol. 1999; 87(6):2032-6. [PubMed: 10601146]

5. Bailey DM, Young IS, McEneny J, Lawrenson L, Kim J, Barden J, Richardson RS. Regulation of free radical outflow from an isolated muscle bed in exercising humans. Am J Physiol Heart Circ Physiol. 2004; 287(4):H1689-99. [PubMed: 15155256]

6. Barreiro E, de la Puente B, Minguella J, Corominas JM, Serrano S, Hussain SN, Gea J. Oxidative stress and respiratory muscle dysfunction in severe chronic obstructive pulmonary disease. Am J Respir Crit Care Med. 2005; 171(10):1116-24. [PubMed: 15735057]

7. Benzie IF, Strain JJ. The ferric reducing ability of plasma (FRAP) as a measure of "antioxidant power": the FRAP assay. Anal Biochem. 1996; 239(1):70-6. [PubMed: 8660627]

8. Borg, G. Borg's Perceived Exertion and Pain Scales. Human Kinetics; Champaign, IL: 1998. p. 44-6.

9. Bradley DW, Emery G, Maynard JE. Vitamin C in plasma: a comparative study of the vitamin stabilized with trichloroacetic acid or metaphosphoric acid and the effects of storage at -70 degrees, -20 degrees, 4 degrees, and 25 degrees on the stabilized vitamin. Clin Chim Acta. 1973; 44(1):4752. [PubMed: 4707637]

10. Celli BR, MacNee W. Standards for the diagnosis and treatment of patients with COPD: a summary of the ATS/ERS position paper. Eur Respir J. 2004; 23(6):932-46. [PubMed: 15219010]

11. Couillard A, Koechlin C, Cristol JP, Varray A, Prefaut C. Evidence of local exercise-induced systemic oxidative stress in chronic obstructive pulmonary disease patients. Eur Respir J. 2002; 20(5):1123-9. [PubMed: 12449164]

12. Couillard A, Maltais F, Saey D, Debigare R, Michaud A, Koechlin C, LeBlanc P, Prefaut C. Exercise-induced quadriceps oxidative stress and peripheral muscle dysfunction in patients with chronic obstructive pulmonary disease. Am J Respir Crit Care Med. 2003; 167(12):1664-9. [PubMed: 12672647]

13. Couillard A, Prefaut C. From muscle disuse to myopathy in COPD: potential contribution of oxidative stress. Eur Respir J. 2005; 26(4):703-19. [PubMed: 16204604]

14. Crecelius AR, Kirby BS, Voyles WF, Dinenno FA. Nitric oxide, but not vasodilating prostaglandins, contributes to the improvement of exercise hyperemia via ascorbic acid in healthy older adults. Am J Physiol Heart Circ Physiol. 2010; 299(5):H1633-41. [PubMed: 20817831]

15. Delliaux S, Brerro-Saby C, Steinberg JG, Jammes Y. Reactive oxygen species activate the group IV muscle afferents in resting and exercising muscle in rats. Pflugers Arch. 2009; 459(1):143-50. [PubMed: 19701650]

16. Donato AJ, Uberoi A, Bailey DM, Wray DW, Richardson RS. Exercise-induced brachial artery vasodilation: effects of antioxidants and exercise training in elderly men. Am J Physiol Heart Circ Physiol. 2010; 298(2):H671-8. [PubMed: 19966056]

17. Donato AJ, Uberoi A, Wray DW, Nishiyama S, Lawrenson L, Richardson RS. Differential effects of aging on limb blood flow in humans. American journal of physiology. Heart and circulatory physiology. 2006; 290(1):H272-8. [PubMed: 16183733]

18. Harris RA, Wray DW, Nishiyama SK, Berkstresser KA, Richardson RS. The Influence of an Antioxidant Cocktail on Vascular Function in COPD. FASEB J. 2008; 22(1235):16.

19. Heunks LM, Vina J, van Herwaarden CL, Folgering HT, Gimeno A, Dekhuijzen PN. Xanthine oxidase is involved in exercise-induced oxidative stress in chronic obstructive pulmonary disease. Am J Physiol. 1999; 277(6 Pt 2):R1697-704. [PubMed: 10600916]

20. Jackson MJ. An overview of methods for assessment of free radical activity in biology. Proc Nutr Soc. 1999; 58(4):1001-6. [PubMed: 10817168]

21. Killian KJ, Leblanc P, Martin DH, Summers E, Jones NL, Campbell EJ. Exercise capacity and ventilatory, circulatory, and symptom limitation in patients with chronic airflow limitation. Am Rev Respir Dis. 1992; 146(4):935-40. [PubMed: 1416421]

22. Kirby BS, Voyles WF, Simpson CB, Carlson RE, Schrage WG, Dinenno FA. Endotheliumdependent vasodilatation and exercise hyperaemia in ageing humans: impact of acute ascorbic acid administration. J Physiol. 2009; 587(Pt 9):1989-2003. [PubMed: 19307300] 
23. Kirkham P, Rahman I. Oxidative stress in asthma and COPD: antioxidants as a therapeutic strategy. Pharmacol Ther. 2006; 111(2):476-94. [PubMed: 16458359]

24. Koechlin C, Couillard A, Simar D, Cristol JP, Bellet H, Hayot M, Prefaut C. Does oxidative stress alter quadriceps endurance in chronic obstructive pulmonary disease? Am J Respir Crit Care Med. 2004; 169(9):1022-7. [PubMed: 15001462]

25. Lepers R, Maffiuletti NA, Rochette L, Brugniaux J, Millet GY. Neuromuscular fatigue during a long-duration cycling exercise. J Appl Physiol. 2002; 92(4):1487-93. [PubMed: 11896014]

26. Mador MJ, Kufel TJ, Pineda LA, Steinwald A, Aggarwal A, Upadhyay AM, Khan MA. Effect of pulmonary rehabilitation on quadriceps fatiguability during exercise. Am J Respir Crit Care Med. 2001; 163(4):930-5. [PubMed: 11282768]

27. McKenna MJ, Medved I, Goodman CA, Brown MJ, Bjorksten AR, Murphy KT, Petersen AC, Sostaric S, Gong X. N-acetylcysteine attenuates the decline in muscle $\mathrm{Na}+\mathrm{K}+-$ pump activity and delays fatigue during prolonged exercise in humans. J Physiol. 2006; 576(Pt 1):279-88. [PubMed: 16840514]

28. Montuschi P, Collins JV, Ciabattoni G, Lazzeri N, Corradi M, Kharitonov SA, Barnes PJ. Exhaled 8-isoprostane as an in vivo biomarker of lung oxidative stress in patients with COPD and healthy smokers. Am J Respir Crit Care Med. 2000; 162(3 Pt 1):1175-7. [PubMed: 10988150]

29. Ochs-Balcom HM, Grant BJ, Muti P, Sempos CT, Freudenheim JL, Browne RW, McCann SE, Trevisan M, Cassano PA, Iacoviello L, Schunemann HJ. Antioxidants, oxidative stress, and pulmonary function in individuals diagnosed with asthma or COPD. Eur J Clin Nutr. 2006; 60(8): 991-9. [PubMed: 16482071]

30. Pinamonti S, Leis M, Barbieri A, Leoni D, Muzzoli M, Sostero S, Chicca MC, Carrieri A, Ravenna F, Fabbri LM, Ciaccia A. Detection of xanthine oxidase activity products by EPR and HPLC in bronchoalveolar lavage fluid from patients with chronic obstructive pulmonary disease. Free Radic Biol Med. 1998; 25(7):771-9. [PubMed: 9823542]

31. Polkey MI, Kyroussis D, Hamnegard CH, Mills GH, Green M, Moxham J. Quadriceps strength and fatigue assessed by magnetic stimulation of the femoral nerve in man. Muscle Nerve. 1996; 19(5):549-55. [PubMed: 8618551]

32. Powers SK, Jackson MJ. Exercise-induced oxidative stress: cellular mechanisms and impact on muscle force production. Physiol Rev. 2008; 88(4):1243-76. [PubMed: 18923182]

33. Powers SK, Nelson WB, Hudson MB. Exercise-induced oxidative stress in humans: cause and consequences. Free radical biology \& medicine. 2011; 51(5):942-50. [PubMed: 21167935]

34. Reid MB, Stokic DS, Koch SM, Khawli FA, Leis AA. N-acetylcysteine inhibits muscle fatigue in humans. J Clin Invest. 1994; 94(6):2468-74. [PubMed: 7989604]

35. Richardson RS, Donato AJ, Uberoi A, Wray DW, Lawrenson L, Nishiyama S, Bailey DM. Exercise-induced brachial artery vasodilation: role of free radicals. Am J Physiol Heart Circ Physiol. 2007; 292(3):H1516-22. [PubMed: 17114239]

36. Richardson RS, Leek BT, Gavin TP, Haseler LJ, Mudaliar SR, Henry R, Mathieu-Costello O, Wagner PD. Reduced mechanical efficiency in chronic obstructive pulmonary disease but normal peak VO2 with small muscle mass exercise. Am J Respir Crit Care Med. 2004; 169(1):89-96. [PubMed: 14500263]

37. Richardson RS, Sheldon J, Poole DC, Hopkins SR, Ries AL, Wagner PD. Evidence of skeletal muscle metabolic reserve during whole body exercise in patients with chronic obstructive pulmonary disease. Am J Respir Crit Care Med. 1999; 159(3):881-5. [PubMed: 10051266]

38. Trinity JD, Groot HJ, Layec G, Rossman MJ, Ives SJ, Runnels S, Gmelch B, Bledsoe A, Richardson RS. Nitric oxide and passive limb movement: a new approach to assess vascular function. J Physiol. 2012; 590(Pt 6):1413-25. [PubMed: 22310310]

39. Wagner PD. Possible mechanisms underlying the development of cachexia in COPD. Eur Respir J. 2008; 31(3):492-501. [PubMed: 18310396]

40. Wheeler CR, Salzman JA, Elsayed NM, Omaye ST, Korte DW Jr. Automated assays for superoxide dismutase, catalase, glutathione peroxidase, and glutathione reductase activity. Anal Biochem. 1990; 184(2):193-9. [PubMed: 2327564] 
41. Wray DW, Nishiyama SK, Harris RA, Zhao J, McDaniel J, Fjeldstad AS, Witman MA, Ives SJ, Barrett-O'Keefe Z, Richardson RS. Acute reversal of endothelial dysfunction in the elderly after antioxidant consumption. Hypertension. 2012; 59(4):818-24. [PubMed: 22353612]

42. Wray DW, Nishiyama SK, Monnet A, Wary C, Duteil SS, Carlier PG, Richardson RS. Antioxidants and aging: NMR-based evidence of improved skeletal muscle perfusion and energetics. Am J Physiol Heart Circ Physiol. 2009; 297(5):H1870-5. [PubMed: 19767527]

43. Wray DW, Uberoi A, Lawrenson L, Bailey DM, Richardson RS. Oral antioxidants and cardiovascular health in the exercise-trained and untrained elderly: a radically different outcome. Clin Sci (Lond). 2009; 116(5):433-41. [PubMed: 18795893]

44. Yagi K. A simple fluorometric assay for lipoperoxide in blood plasma. Biochem Med. 1976; 15(2): 212-6. [PubMed: 962904] 


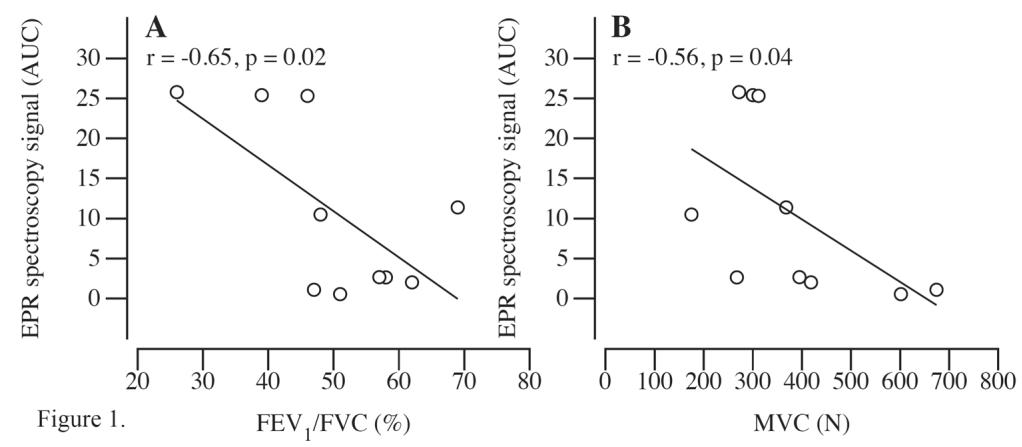

Figure 1. Relationships between the forced expiratory volume in 1 second/forced vital capacity ratio $\left(\mathrm{FEV}_{1} / \mathrm{FVC}\right)$ and maximal voluntary quadriceps contraction (MVC) with resting free radical concentration assessed by electron paramagnetic resonance (EPR) spectroscopy Resting free radical concentration (AUC, arbitrary units) was moderately inversely correlated with $\mathrm{A}: \mathrm{FEV}_{1} / \mathrm{FVC}$ and $\mathrm{B}$ : baseline quadriceps $\mathrm{MVC}$. $* \mathrm{p}<0.05$, one-tailed test. 

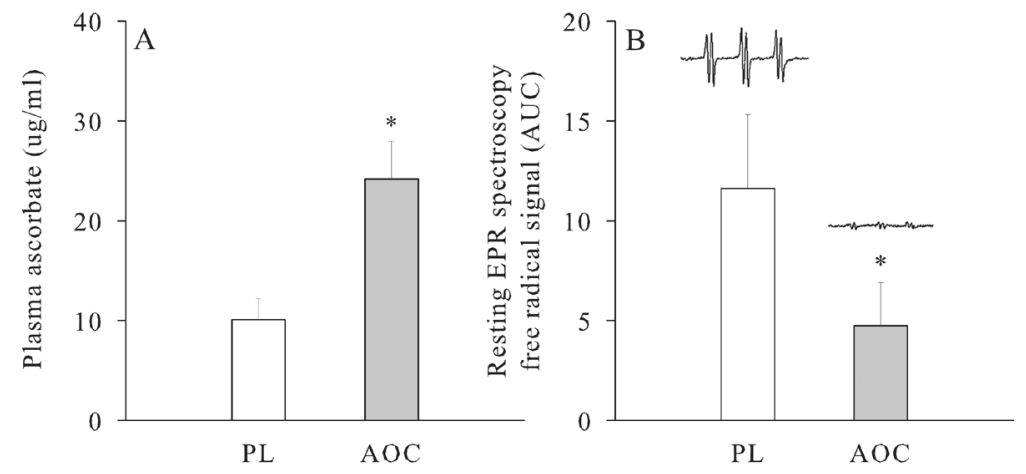

Figure 2. Resting antioxidant and oxidant status assayed in the plasma following placebo (PL) and antioxidant cocktail (AOC) consumption

Data are presented as mean \pm S.E.M. AOC consumption resulted in a $140 \%$ increase in plasma ascorbate (A) and a $63 \%$ reduction in the free radical concentration assessed by electron paramagnetic resonance (EPR) spectroscopy (presented with representative spectroscopy signal) and expressed as the area under the curve (AUC, arbitrary units) (B). * Significantly different from placebo condition, $\mathrm{p}<0.05$. 

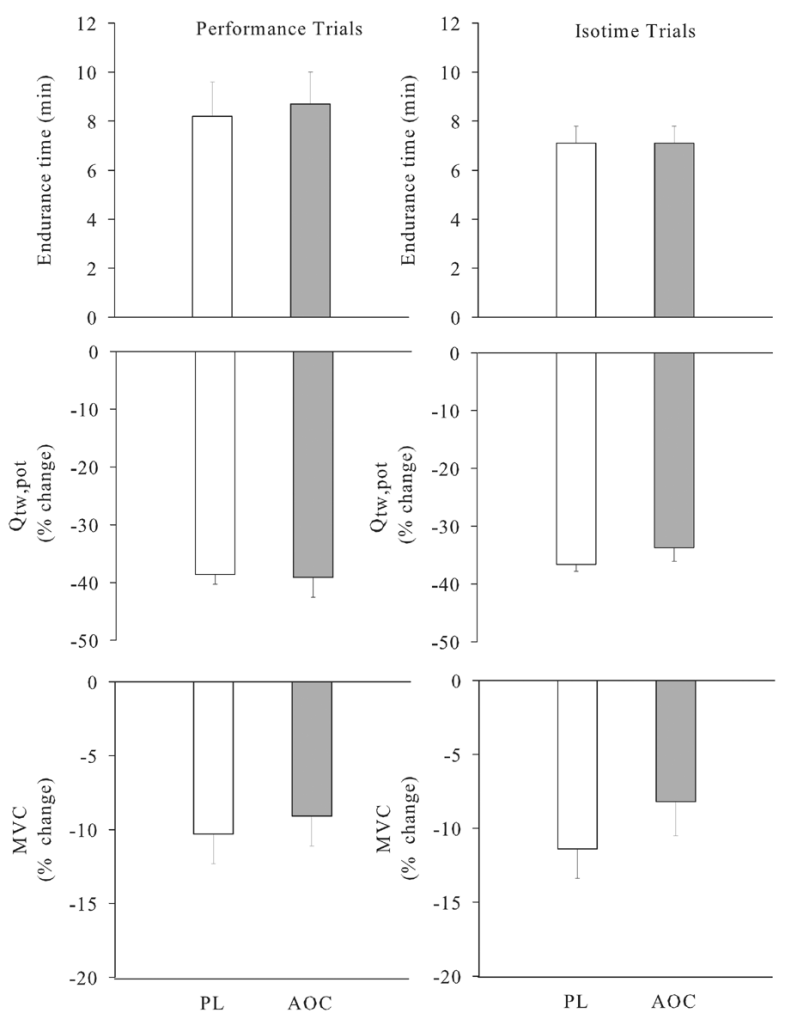

Figure 3. Endurance time to exhaustion and end-exercise quadriceps fatigue assessed following constant-load knee extensor exercise to exhaustion (performance trials) and following exercise matched for intensity $(23 \pm 3 \mathrm{~W})$ and duration (isotime trials) with either the consumption of a placebo (PL) or an antioxidant cocktail (AOC)

Data are presented as mean \pm S.E.M. Quadriceps fatigue values represent the percent change from pre- to postexercise. $\mathrm{Q}_{\mathrm{tw}}$,pot, potentiated twitch force; MVC, maximal voluntary contraction. There were no significant differences. 

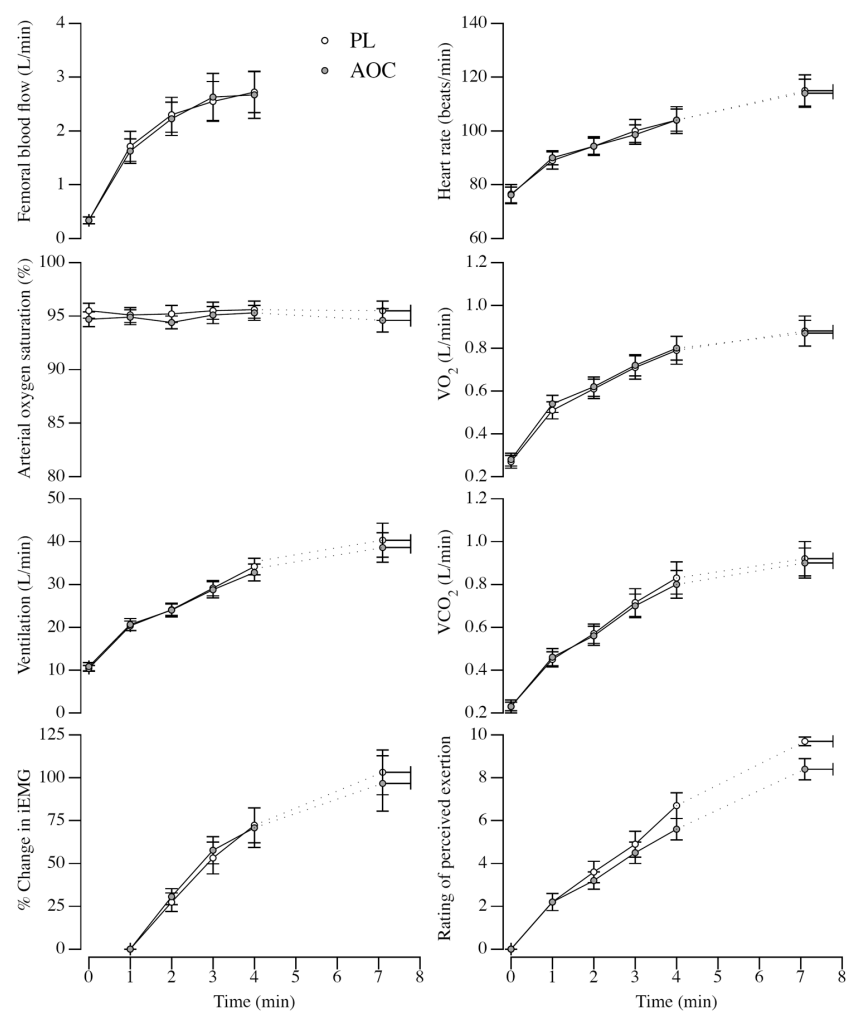

Figure 4. Physiological responses to constant workload isotime knee extensor exercise matched for intensity $(23 \pm 3 \mathrm{~W})$ and duration $(7.1 \pm 0.7 \mathrm{~min})$ following consumption of either a placebo (PL) or an antioxidant cocktail (AOC)

Group mean data ( \pm S.E.M) over the first four minutes of exercise, which were attained by all subjects. The final time point represents end-exercise values. End exercise values for femoral blood flow are not reported due to loss of signal. VE, ventilation; $\mathrm{VO}_{2}$, oxygen consumption; $\mathrm{VCO}_{2}$, carbon dioxide production; $\mathrm{iEMG}$, integrated electromyogram from the vastus lateralis. There were no significant differences between interventions. 


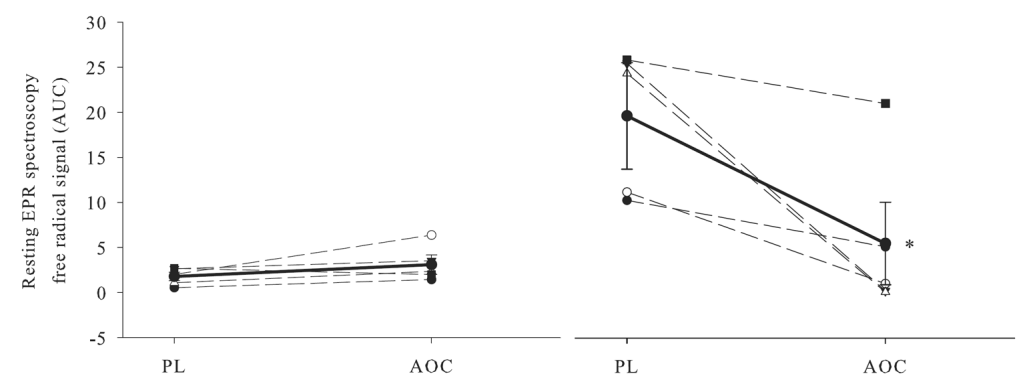

Figure 5. Individual chang es in the a-Phenyl-tert-butylnitrone (PBN) spin adduct area under the curve (AUC) assessed by electron paramagnetic resonance (EPR) spectroscopy following the ingestion of both the placebo (PL) and antioxidant cocktail (AOC)

Individual responses have been separated into two groups, those with low (left) and high (right) basal free radical levels in the PL condition. As illustrated, the AOC appeared to only have an effect in those with high basal free radical levels, evidence that the baseline free radical load influences the efficacy of the AOC. *Significantly different from placebo condition, $\mathrm{p}<0.05$. 


\section{Table 1}

\section{Subject characteristics}

\begin{tabular}{lc}
\hline Age $(\mathrm{yr})$ & $62 \pm 3$ \\
Height $(\mathrm{m})$ & $1.73 \pm 0.03$ \\
Weight $(\mathrm{kg})$ & $84.1 \pm 7.4$ \\
BMI $\left(\mathrm{kg} / \mathrm{m}^{2}\right)$ & $27.9 \pm 1.7$ \\
Quadriceps muscle mass $(\mathrm{kg})$ & $1.71 \pm 0.2$ \\
Peak knee-extensor work rate (W) & $28 \pm 3$ \\
Male/Female & $7 / 3$ \\
Pulmonary function & \\
Forced vital capacity, 1 (\% predicted) & $3.6 \pm 0.2(86.2 \pm 4.7)$ \\
Forced expiratory volume in one s, $1 / \mathrm{s}(\%$ predicted) & $1.8 \pm 0.2(57.1 \pm 4.6)$ \\
FEV ${ }_{1} /$ FVC (\%) & $50.4 \pm 4.9$ \\
Resting arterial blood gases ${ }^{\dagger}(\mathbf{n}=\mathbf{9})$ & \\
Hemoglobin concentration $(\mathrm{g} / \mathrm{dl})$ & $13.5 \pm 0.5$ \\
Oxyhemoglobin $(\%)$ & $91.9 \pm 0.6$ \\
Partial pressure of oxygen (mmHg) & $69.9 \pm 2.0$ \\
Partial pressure of carbon dioxide $(\mathrm{mmHg})$ & $32.2 \pm 2.0$ \\
Bicarbonate (mmol/l) & $22.0 \pm 1.4$ \\
pH & $7.45 \pm 0.01$ \\
\hline
\end{tabular}

Values expressed as mean \pm S.E.M. FEV1/FVC $=$ Forced expiratory volume in one second relative to forced vital capacity. BMI $=$ Body mass index.

Arterial blood gases were obtained from 9 subjects during a separate study.

${ }^{\dagger}$ Note: Study performed at $1400 \mathrm{~m}$. 